\title{
Communications
}

\section{Rappel volontaire}

Produit: KIOVIG solution pour perfusion

$(30 \mathrm{~g} / 300 \mathrm{ml})$

Numéro d'article: 1502548

Lot: LE12P035AD

Numéro d'autorisation: 57469

La société Baxter Suisse SA vous informe par la présente du rappel volontaire du lot KIOVIG LE12P035AD. Cette mesure de prévention a été prise en raison d'une augmentation de l'incidence de réactions d'hypersensibilité après l'utilisation du lot sus-mentionné. Seul le lot sus-mentionné est concerné par ce rappel.

Nous vous prions donc de ne plus utiliser KIOVIG $(30 \mathrm{~g} / 300 \mathrm{ml})$ portant la désignation de lot LE12P035AD, de séparer de vos stocks toutes les unités restantes de ce lot et de les marquer comme bloquées.

Si vous avez livré ce lot à d'autres fournisseurs ou institutions, veuillez transmettre la présente information immédiatement aux interlocuteurs concernés.

Ce rappel volontaire a lieu en accord avec l'Institut suisse des produits thérapeutiques, Swissmedic.

Si vous avez des questions, veuillez appeler le numéro suivant: 04490852 17. Vous pouvez également nous joindre par courriel à l'adresse: Switzerland_SHS_CQA[at]baxter.com.

Nous vous prions d'annoncer tous effets indésirables liés à l'utilisation de KIOVIG à un des centres régionaux de pharmacovigilance à l'aide du formulaire Swissmedic correspondant (www.swissmedic.ch).

Nous déplorons les désagréments occasionnés par ce rappel et vous remercions en même temps de nous aider à mettre en œuvre cette préventive mesure de sécurité.

Baxter SA, Volketswil

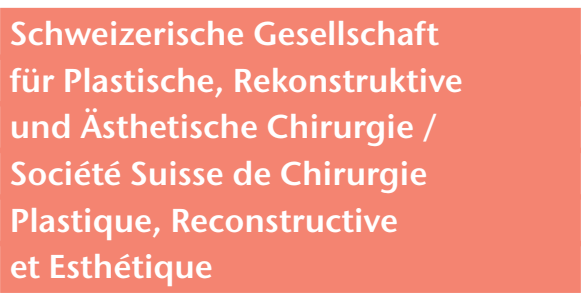

Vorstand / Comité (ab 1.10.2014 für 2 Jahre/à partir du 1.10.2014 pour deux ans)

Die Mitgliederversammlung der Schweizerischen Gesellschaft für Plastische, Rekonstruktive und Ästhetische Chirurgie hat am 5. September 2014 folgenden Vorstand gewählt bzw. bestätigt:
L'Assemblée générale de la Société Suisse de Chirurgie Plastique, Reconstructive et Esthétique du 5 septembre 2014 a élu respectivement confirmé le comité suivant:

\section{Präsidentin/Présidente \\ Dr Pierre Quinodoz}

Pastpräsidentin/Présidente sortante

Prof. Dr Brigitte Pittet-Cuénod, Genève

Vizepräsident/Vice-président

Dr. med. Yves Brühlmann, Bern

\section{Kassier/Trésorier}

Prof. Dr. med. Mihai A. Constantinescu

\section{Mitglieder/Membres}

Dr. med. Thomas Fischer, Bern

Prof. Dr. med. Jörg Grünert, St. Gallen

Dr Patricia Roggero, Lausanne

Prof. Dr. med. Dirk J. Schaefer, Basel

Sujets actuels de forum
Ursula Zybach, Présidente exécutive Santé publique Suisse, membre du Grand Conseil
du canton de Berne
Manifeste suisse relatif à la Public Mental Health
Quels sont vos revendications et souhaits concrets?

